\title{
Unilateral medial forebrain bundle activation selectively enhances conditioned orienting head turns and ipsilateral cingulate cortex evoked field responses in cats
}

\author{
MARKKU PENTTONEN, TAPANI KORHONEN, JUHA ARIKOSKI, and TIMO RUUSUVIRTA \\ University of Jyväskylä, Jyväskylä, Finland \\ and \\ KENNETH HUGDAHL \\ University of Bergen, Bergen, Norway
}

\begin{abstract}
Effects of a unilateral medial forebrain bundle (MFB) stimulation unconditioned stimulus (US) on conditioned head turn and bilateral cingulate cortex field potentials were studied in cats. Conditioned stimuli (CSs) of different frequences were given randomly to either ear. The CS + was followed by the US, and the CS - was presented alone. Before conditioning most cats predominantly turned toward the ear to which the CSs were presented, whereas after conditioning the head turns were in one direction, most prominently contralateral to the US. Negative field potentials were greater in the cingulate cortex ipsilateral to the US than in the cingulate cortex contralateral to the US. Cross correlations indicated similarities between the waveforms recorded for the CS and the US within each cingulate cortex, but differences between the two sides of the cingulate cortex. It is suggested that the unilateral MFB system has brain side specific effects on conditioned orienting head turns and cingulate cortex neuronal activity.
\end{abstract}

The primary aim of the present study was to determine whether unilateral conditioned stimulus (CS) and unconditioned stimulus (US) presentations would result in lateralized orienting head movements and cingulate cortex neuronal activity during differential classical conditioning in the cat. Although the nictitating membrane studies in the rabbit have suggested that the memory trace for the nictitating membrane conditioned response (CR) is stored unilaterally in the cerebellum or its deep structures (Thompson, 1986), brain side specific effects of classical conditioning have not been extensively studied at other brain levels and with other classical conditioning procedures.

In previous studies, cats showed a persistent tendency to turn their heads toward the source of the tone when the tone CS was presented to one ear and paired with electrical stimulation of the medial forebrain bundle (MFB) in the lateral hypothalamus (Korhonen \& Penttonen, 1989a, 1989b). These head-turn CRs disappeared during subsequent unpaired presentations of the CS and US. If, however, the training consisted only of unpaired presentations of the CS and US, the head-turn CRs did not appear at all. Instead, the cats showed orienting movements

This research was supported by the Finnish Academy. Correspondence should be addressed to Markku Penttonen, Department of Psychology, University of Jyväskylä, P.O. Box 35, SF-40351 Jyväskylä, Finland (e-mail: penttone@jyu.fi). only during the first few CS presentations before complete habituation. The head-turn CR was, furthermore, shown to be specific to the side of the tone presentation in a recent differential conditioning study (Penttonen, Korhonen, Arikoski, \& Hugdahl, 1993). When a tone presented to one ear was paired with MFB stimulation (CS+) and the same tone presented to the opposite ear was not paired with stimulation (CS-), the cats showed larger head turns to the CS + than to the CS - and turned toward the side of the CS + tone not only on CS + trials but also, although with a smaller amplitude, on CS - trials. The head-turn CR was not related to the head-turn unconditioned response (UR) elicited by the MFB stimulation. This was controlled by presenting the $\mathrm{CS}+$ to the ear contralateral to the direction of the UR.

The aim of the present study was to determine whether the head-turn CR is an enhancement of an orientation toward the CS or whether it results from unilateral effects of the US. For this purpose, tones of different frequencies were used as $\mathrm{CS}-$ and $\mathrm{CS}+$. The CS + tone, which was paired with an MFB stimulation US, was randomly presented to either the left or the right ear. The $\mathrm{CS}$ - tone was also presented to each ear in random order, but without the US. The question was whether the cats would develop head-turn CRs toward the ear to which the CS tone was presented on a particular trial or, alternatively, whether they would produce head movements biased in one direction independently of the actual side of 
tone presentation. A more specific question was to determine whether the frequency of head-turn CRs ipsi- and contralateral to the US is different. Unilateral head-turn CRs would imply the brain side specific effect of a unilateral US on learning. To standardize the effect of the stimulation side, all the US electrodes were implanted in the right MFB.

Cingulate cortex field responses were recorded bilaterally to determine the effect of the MFB stimulation US on cortical neuronal activity and to determine whether conditioning-related neuronal changes differ in the cingulate cortex ipsi- and contralateral to the US. Although there is no specific evidence that the cingulate cortex is necessary for head movement conditioning, the current emphasis on multiple forms of learning and memory (Lavond, Kim, \& Thompson, 1993) and also on the contribution of different brain structures at different levels on conditioned responding (Berger, Bassett, \& Orr, 1991) motivated the simultanous recording of head movements and cingulate cortex neuronal activity.

A practical reason for positioning the recording electrodes in the cingulate cortex was its location at the midline of the brain, which simplified the implantation of bilaterally symmetric electrodes. An additional reason for choosing the cingulate cortex for neuronal analysis was that it has been implicated in the initiation of the differentially conditioned rabbit avoidance response (Stolar, Sparenborg, Donchin, \& Gabriel, 1989). It was therefore of interest to determine whether conditioning with an appetitive US would also affect cingulate cortex neuronal activation, which would imply the involvement of the cingulate cortex in conditioned approach response initiation. The analysis of conditioned approach responses should be of particular importance, since most current biological model systems are focused on the analysis of aversively motivated behaviors (Schreurs, 1989).

\section{METHOD}

\section{Animals}

The animals were 13 adult cats weighing $3.2-4.2 \mathrm{~kg}$ at the time of surgery. They were housed in groups and had free access to food and water. A 13:12-h light:dark cycle (lights on at 0800 hours) was employed.

\section{Surgery}

The recording electrodes were made of Trimel-insulated nichrome wire of $50 \mu \mathrm{m}$ diameter, with the recording tip of the wire cut transversely with scissors. The bipolar brain-stimulation electrodes were made of two Teflon-insulated $250-\mu \mathrm{m}$ stainless steel wires. Both tips were separated by approximately $500 \mu \mathrm{m}$ and were exposed to $200-400 \mu \mathrm{m}$.

Under sodium pentobarbital anesthesia $(40 \mathrm{mg} / \mathrm{kg}$ ), two recording electrodes were implanted symmetrically in the left and right cingulate cortex (A5.0, L0.7, H11.0; A4.0, L0.9, H11.0). Two interconnected screws (A20.0, L5.0) were fitted on each side of the skull and served as the indifferent reference electrode for the monopolar recordings. Three stimulation electrodes were aimed at the right MFB at the level of the lateral hypothalamus (A8.5, L2.5,
H-3.0; A9.5, L2.5, H-4.0; and A10.5, L2.5, H-5.0). All the electrodes were connected to two 15-pin D-type connectors, which were attached with anchoring screws and dental acrylic to the skull.

\section{Apparatus and Behavioral Training}

Throughout the behavioral and field potential measurements, the cats were kept in a ventilated, sound-attenuating, and electrically shielded box $(60 \times 48 \times 58 \mathrm{~cm})$ located in a room adjacent to that which housed the control and recording equipment. The door of the box was equipped with a window, and a video camera was placed in front of the box. The room was dark, but the box was illuminated by eight 5 -W light bulbs on the ceiling of the box. The animals were observed during the experiments through a video monitor, and trials were recorded on videotape for the analysis of movements. Two miniature loudspeakers were mounted on a holder attached to a socket in the dental acrylic on the skull. The loudspeakers were positioned symmetrically approximately $2.0 \mathrm{~cm}$ in front of the left and right ears of the cat. The $1000-$ and $2500-\mathrm{Hz}$ CS tones $(65 \mathrm{~dB}$, measured at a distance of $2 \mathrm{~cm}$ ) were delivered through these loudspeakers. The head movements were recorded with a customdesigned three-dimensional acceleration transducer based on piezoelectric elements. The sensitivity of the transducer was $10 \mathrm{mV} / \mathrm{g}$.

Low-noise preamplifiers with a gain of 10 (Analog Devices, AD524) were connected directly to the connector in the acrylic mass on the head of the cat. After this stage, the neural signals were further amplified (gain 400) and filtered $(0.2-50 \mathrm{~Hz})$ using separate equipment and were then fed to an $A / D$ converter (Data Translation 2831G). A flexible, shielded cable connected the animal to the amplifiers and the stimulator.

A microcomputer delivered the discrete trials and randomized the intervals between trials. The computer controlled the operation of the video recorder and the signal generators (Wavetek 148) for the delivery of CS tones. The computer also generated the brainstimulation pulse trains with an optoisolated D/A converter. The trial identification data generated by the microcomputer were displayed on a video monitor.

One week after surgery, the effect of the US was tested. The US consisted of electrical stimulation of the MFB at a pulse width of $0.5 \mathrm{msec}$ (bipolar pulses), a pulse frequency of $100 \mathrm{~Hz}$, and a train duration of $500 \mathrm{msec}$. The electrode that induced orienting and approach movements, usually accompanied by salivation without aversive effects, was selected as the stimulation electrode. Stimulation intensities ranged from 140 to $420 \mu \mathrm{A}$. After selection of the brainstimulation electrode the animals were given an opportunity to adapt to the experimental box for 2 days.

The behavioral training began with a CS test session during which 1000 - and $2500-\mathrm{Hz}$ tones of $1,500-\mathrm{msec}$ duration were delivered at equal intensity to either the left or the right ear in random order. The total number of trials was 80 and, due to randomization, each tone was presented approximately 20 times to the left ear and approximately 20 times to the right ear. Trials were presented at a mean intertrial interval of $30 \mathrm{sec}$ (range $20-40 \mathrm{sec}$ ). This phase of the experiment was included in order to reveal the existence of any differences in orienting movements and/or their habituation to the CS tones.

Differential conditioning began on the second day and continued for 4 days. One randomly selected tone (either 1000 or $2500 \mathrm{~Hz}$ ) was used as the CS + and the other as the CS- In the CS - trials, the $1500-\mathrm{msec}$ tone was presented to either the left or the right ear. In the CS+ trials, the alternative 1,500 -msec tone was presented to either the left or the right ear, overlapping during the last $500 \mathrm{msec}$ with the US. Each daily session comprised 80 trials. As a result of randomization, both the $\mathrm{CS}-$ and $\mathrm{CS}+$ tones were presented approximately 20 times to the left ear and 20 times to the right ear. The intertrial intervals were randomized over a range of $20-40 \mathrm{sec}$ (mean $30 \mathrm{sec}$ ). The final phase of the training, the US 
test session, consisted of 40 electrical brain-stimulation US presentations over a range of $40-80 \mathrm{sec}$ (mean $60 \mathrm{sec}$ ).

\section{Histology}

After the experiments, the animals were anesthetized with a lethal dose of sodium pentobarbital and perfused via the carotic arteries with physiological saline followed by $10 \%$ formalin. After marking the electrodes by passing $10 \mu \mathrm{A}$ direct current for $20 \mathrm{sec}$, the brain was removed from the skull. The brain, together with the electrode assembly, was kept in formalin for a week. The electrodes were removed, and frozen $80-\mu \mathrm{m}$ cross sections of the brain were cut, mounted on glass slides, and stained with cresyl violet. The locations of the electrode tips were then compared against the stereotaxic atlas (Snider \& Niemer, 1961).

\section{Data Reduction and Statistical Analysis}

The qualitative aspects of the cats' behavior were evaluated from the video recordings. Orienting movements to the CSs presented to each ear during the CS test, CRs to the CSs presented to each ear during the final conditioning session, and URs to the brainstimulation USs during the US test were all monitored on the video recorder. Field potential and movement signals were digitized at the rate of 200 samples per second with an IBM PC/AT compatible microcomputer.

Signal analysis was based on a 2,000-msec period, which included 500-msec preCS, 1,000-msec CS, and 500-msec US periods. Four separate averages were computed for the $\mathrm{CS}-$ and $\mathrm{CS}+$ tones presented to each ear during the CS test session and the conditioning sessions, and one average was computed for the US trials of the US test session. Movement signals were rectified before averaging in order to obtain a unidimensional index for the acceleration of the head. A baseline correction was performed by subtracting the average of the preCS samples from each movement signal and neural recording point for the observed period.

The numerical indices used in the statistical analyses were formed by computing an average for the values between 125 and $325 \mathrm{msec}$ after the tone onset. The averaged curves showed a clear negative deflection in most field potential recordings during this period. Movement and evoked responses were analyzed for 12 animals only, due to recording artifacts in one animal.

The analysis of the head-movement data was based on a $2 \times 2 \times 4$ randomized block design (Kirk, 1968) with a conditioning factor (CS- vs. CS+), tone laterality factor (ipsilateral vs. contralateral to the US), and a training sessions factor (four daily sessions). All three factors were treated as within-subjects factors. For the evoked responses, a hemisphere factor (cingulate cortex ipsilateral vs. contralateral to the US) was also included to form a $2 \times 2 \times 2 \times 4$ randomized block design. Further analyses were performed for the CS test session by reducing the designs so that they did not include the sessions factor. Geisser-Greenhouse epsilon-corrected degrees of freedom were applied to the analysis of the within-subjects factors to adjust for possible heterogeneity of covariances. Significant main and interaction effects were follow-up analyzed with Tukey's HSD test (Kirk, 1968; $p<.05$ ).

\section{RESULTS}

\section{Head Movement}

During the CS test session, when the CS - and CS + tones were presented to each ear in random order, all the cats initially moved their heads in response to the tones, although these responses quickly habituated. The headmovement response was considered habituated if it was not observed during three consecutive tone presentations.
On average, this criterion was achieved after 6.5 tones to the left ear and 5.5 tones to the right ear. The difference between ears was not statistically significant. Analysis of the direction of the head movements before habituation showed that 11 out of 13 cats predominantly turned their heads toward the source of the tone. Two cats showed a dominant tendency to turn to the right regardless of the ear to which the tone was delivered.

During conditioning, all the cats developed headmovement CRs. Although these CRs already appeared during the first session, they stabilized gradually during subsequent sessions. During the final conditioning session, the CRs to the CS + presentations constituted rapid and vigorous head turns, unaccompanied by any other body movements. After each trial, the cat slowly resumed a typical forward-looking head position.

Only 3 cats showed head-turn CRs in the actual direction of the CS + tones, even though the tendency to turn toward the tone source was observed in 11 out of 13 cats during the CS test session. Furthermore, the same 2 cats that had preferred to turn to the right during the CS test session also showed a head-turn CR to the right. Of the remaining 8 cats, 2 cats generally turned ipsilateral and 6 turned contralateral to the US in response to the CS+ presentations, regardless of the ear to which the tone was presented.

The cats also moved their heads in response to CStones; however, in general, these movements had a longer onset latency, they were slower, and the displacement of the head was not as great as that to the CS + tones. The direction of the head turn to the CS - was the same as that to the CS + . Thus, 3 cats turned toward the tone during both the $\mathrm{CS}-$ and the CS + trials, and 10 cats turned in one preferred direction in response to both the CSand the CS + tones. In the latter animals, $95 \%$ of the head turns, on average, were in the preferred direction.

The head-movement UR evoked by the US was not related to the head-turn CR. A head-turn UR to the left was observed in 3 cats; in 1 of these cats, the head-turn CR was also to the left, but in 1 other cat it was to the right. A head-movement UR upward or forward was found in 10 cats, 2 of which also showed a slight bias to the right.

Analysis of variance of head-movement acceleration revealed significant effects of conditioning $[F(1,11)=10.73$, $p<.01]$ and session $[F(1,14)=9.16, p<.01]$ and a significant conditioning $\times$ session interaction $[F(2,23)=$ $8.93, p<.01$ ]. Further analysis of the interaction effect with Tukey's HSD test showed that head accelerations did not differ in response to the CS - and the CS + during the first session, but that they were greater to the CS + than to the CS - from the second to the final session (Figure 1). Futhermore, in response to the CS-, there were no differences in head acceleration between sessions; however, in response to the $\mathrm{CS}+$, they were greater from the second to the final session than during the first session and greater during the final session than during second session. No statistically significant effects were found for 


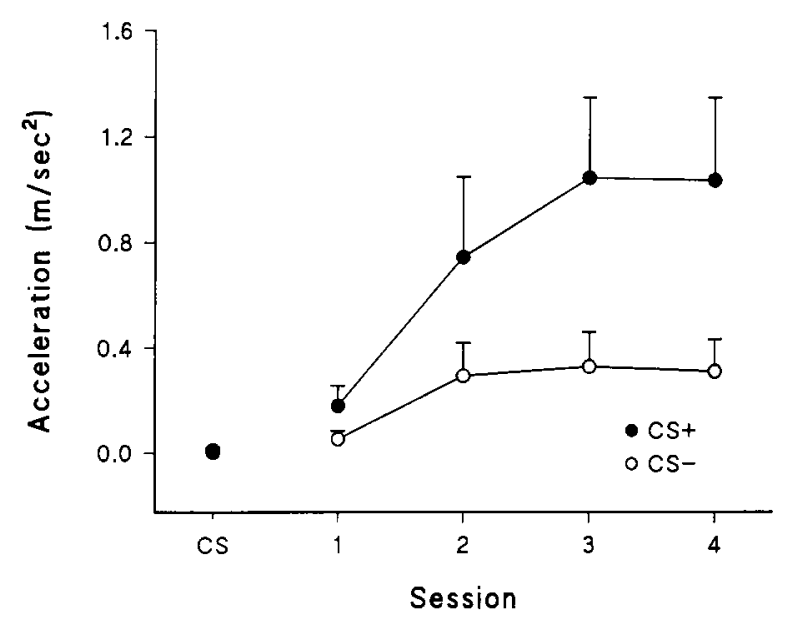

Figure 1. Changes in acceleration of head movements in the 125to 325-msec range in response to $\mathrm{CS}-$ and $\mathrm{CS}+$ tones during the CS test session and the four discriminative conditioning sessions. Bars indicate $S E M$ for 12 cats.

the tone laterality factor. An additional analysis of variance computed for the CS test session did not reveal any significant effects.

Analysis of variance performed on movement onset latencies revealed significant effects of conditioning $[F(1,10)=$ $13.22, p<.01]$ and session $[F(1,16)=9.91, p<.01]$. Average onset latency was shorter for CS+ tones (214 msec) than for CS - tones (396 msec). Tukey's HSD analysis showed that a significant decrease in onset latencies occurred between the first and the final sessions $(425,320,239$, and $236 \mathrm{msec}$, respectively).

\section{Cingulate Cortex Field Potential Responses}

Cingulate cortex field potential responses recorded ipsiand contralateral to the brain-stimulation US are shown for 1 cat in Figure 2. In these, as in most other recordings, a negative field potential response started $25-50 \mathrm{msec}$ after the tone onset. This initial negative potential was often masked, as in the contralateral recording of the last session, by a positive field response that peaked at about $75 \mathrm{msec}$. The negative field potential response later formed a broad wave that peaked at about $260 \mathrm{msec}$ after the tone onset. The broad negative field potential response usually ended at $400-500 \mathrm{msec}$ after the tone onset, and, in most cases, a broad positive potential followed, lasting until the beginning of the US.

The broad negative field potential response alone was associated with the experimental manipulations and, in general, a mean of the values between 125 and $325 \mathrm{msec}$ after the tone onset represented these changes most reliably. Figure 2 shows that the negative field potential response was greater in the cingulate cortex ipsilateral than contralateral to the brain stimulation US, and that, during the final conditioning session, a greater difference in negative field potential in response to the CS - and the $\mathrm{CS}+$ was found in the ipsilateral relative to that found

in the contralateral cingulate cortex. The large field potential response at the end of the CS + recordings demonstrates that a negative field potential in the cingulate cortex also appeared in response to the US. Finally, it can be seen that the beginning of the negative field response preceded the head movements. An analysis of the relation between evoked response and head-movement changes over trials was not, however, possible in the present study since, due to their variability, the individual evoked responses had to be averaged over all trials in each session. At the session level, however, it was apparent that even though both the field response and movement changes appeared during the first conditioning session, neither became prominent until the second session.

Analysis of variance involving the amplitude of the broad negative field potential response showed significant effects of session $[F(1,17)=6.12, p<.05]$ and hemisphere $[F(1,11)=7.51, p<.05]$. The conditioning main effect approached statistical significance $[F(1,11)=3.57$, $p<.10]$. In addition, significant effects of conditioning $\times$ hemisphere interaction $[F(1,11)=9.06, p<.05]$ and session $\times$ hemisphere interaction $[F(1,11)=6.96, p<$ $.01]$ appeared. Further analysis of the significant session $x$ hemisphere interaction effects with Tukey's HSD test revealed that there were no differences between the contralateral and ipsilateral cingulate cortex recordings during the first conditioning session. During subsequent sessions, the negative field potential response was greater in the ipsilateral than in the contralateral cingulate cortex. An analysis of the conditioning $\times$ hemisphere inter-

\section{Cingulate cortex}

\section{Contralateral Ipsilateral}
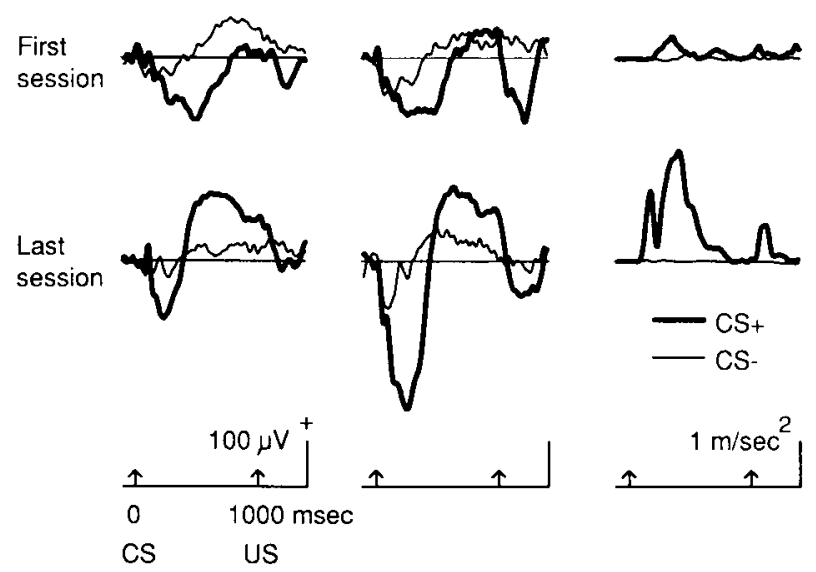

Figure 2. Averaged field potential responses in the cingulate cortex and head-movement transducer responses for 1 cat recorded in response to $\mathrm{CS}-$ and $\mathrm{CS}+$ tones. Cingulate cortex recordings ipsiand contralateral to the site of the MFB stimulation US are shown for the first and final conditioning sessions. The thin line represents CS - , and the thick line represents CS + responses (daily averages of $\mathbf{4 0}$ trials). Arrows indicate CS and US onsets. Negativity is down. 
action effects showed that there were no differences in response to the $\mathrm{CS}-$ and the $\mathrm{CS}+$ in the contralateral cingulate cortex, although the negative field response was greater in response to the $\mathrm{CS}+$ than to the $\mathrm{CS}-$ in the ipsilateral cingulate cortex (Figure 3). Thus, in the ipsilateral cingulate cortex, the negative field response increased over sessions and was larger in response to the $\mathrm{CS}+$ than to the CS - . To control the effect of the direction of the head movement on the observed differences between the ipsi- and contralateral cingulate cortex, an analysis of variance was computed by using the direction of the head-turn CR as an additional between-subjects factor. No differences were observed between cats with an ipsi- or contralateral head-turn CR.

To investigate the effect of the brain-stimulation US on the formation of conditioned field potential responses, the relationship between field potentials recorded in response to the US and CS + was analyzed in 9 animals, from whom artifact-free ipsi- and contralateral cingulate cortex recordings were obtained in response to the brain stimulation. The 3 animals excluded from the analysis had grounding problems. After reducing brain-stimulation artifacts with a digital lowpass filter $(20 \mathrm{~Hz}$ cut-off frequency), correlations were computed between the US and $\mathrm{CS}+$ averages. For this purpose, $400-\mathrm{msec}$ periods were extracted from the US-only averages of the US test session and from the CS + averages of the final paired conditioning session. For the 9 animals, a statistically significant correlation $(p<.01)$ between the US and CS + field potentials was obtained in 15 out of 18 correlations computed for the contra- and the ipsilateral cingulate cortex. The correlations were, on average, .67 and .61 for the contra- and the ipsilateral cingulate cortex and did not differ statistically.

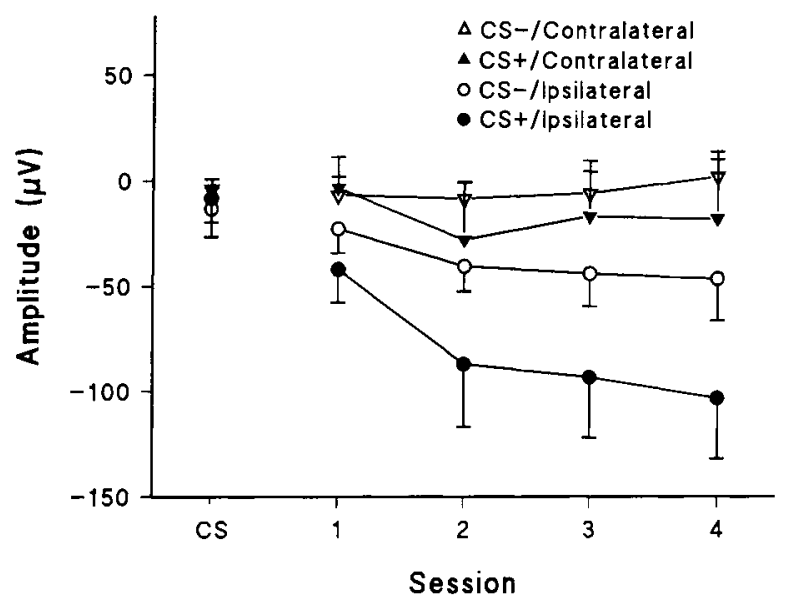

Figure 3. Mean amplitude of negative field potentials in the 125 to 325-msec range in the cingulate cortex recordings in response to $\mathrm{CS}-$ and $\mathrm{CS}+$ tones. Daily averages of $\mathbf{4 0}$ trials during the $\mathrm{CS}$ test session and over the four discriminative conditioning sessions are shown for the cingulate cortex contra- and ipsilateral to the site of the MFB stimulation US. Bars indicate SEM for 12 cats.
Contralateral

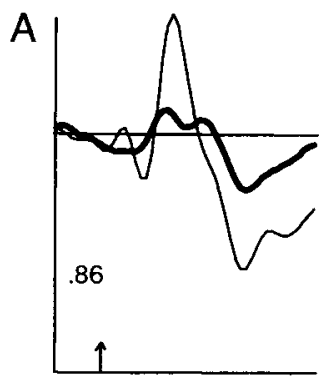

Ipsilateral
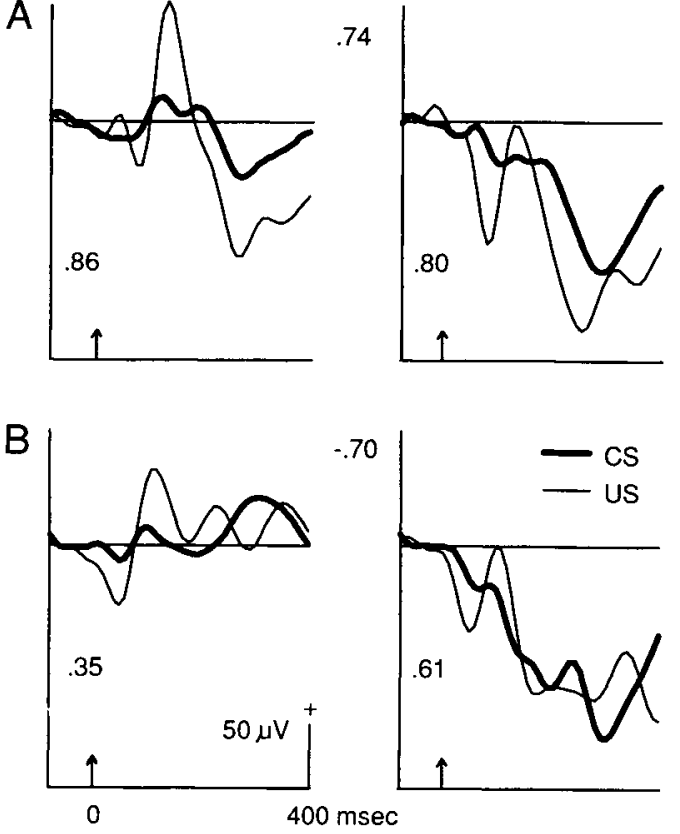

Figure 4. Averaged field potential responses to CS + tone during the final paired conditioning session and to the MFB stimulation US during the US-only test session. The thick line represents responses to the $\mathrm{CS}+$, and the thin line represents responses to the US. Cingulate cortex averages ipsi- and contralateral to the site of the US are shown for 1 cat with a positive (A) and for one cat with a negative (B) correlation between ipsi- and contralateral $\mathrm{CS}+$ responses. Correlations between $\mathrm{CS}+$ and US averages are shown within panels, and correlations between ipsi- and contralateral CS+ averages are shown between left and right panels. Each correlation was based on 80 data sample pairs representing a $400-m s e c$ recording. All correlations are statistically significant $(p<.01)$. Arrows indicate CS and US onsets. Negativity is down.

To study the relationship between the field potentials recorded in the two cingulate cortices, additional correlations were computed between the contra- and the ipsilateral cingulate cortex CS + averages. In 5 out of 9 cats, the correlations between the two cingulate cortex CS + averages were positive and statistically significant $(p<$ .01 ), indicating a similarity between the two sides. In 4 cats, however, the correlations were negative, with statistically significant correlations in 2 cats, indicating a difference between the two sides.

The relation between the CS + averages of the final paired conditioning session and the US-only averages of the US test session is illustrated in Figure 4. US and CS + averages are shown for 1 animal (A) with a statistically significant positive correlation between the ipsi- and contralateral CS + averages, and 1 animal (B) with a negative correlation. The similarity of the US and CS + field potential responses is obvious in both the ipsi- and the contralateral cingulate cortex. This indicates that the form of 
the field potential responses to the CS + closely corresponded to the form of the field potential responses to the brain-stimulation US. Specifically, both cats showed a broad negative field potential response in the ipsilateral cingulate cortex. The same response pattern, although with a smaller amplitude, is also evident in the contralateral recordings for the cat with the positive correlation between the ipsi- and the contralateral cingulate cortex. A positive rather than a negative field potential response is, however, apparent in the contralateral recordings of the cat with a negative correlation between the ipsi- and contralateral CS + averages. Considering all the animals analyzed, in the ipsilateral cingulate cortex, the US evoked negative potentials and these negative potentials (even including their waveforms) were recorded as field potential responses to the CS+ during the final conditioning session. On the other hand, in the contralateral cingulate cortex, the US induced a smaller negative field response and even a positive one; both of these were also recorded during the final conditioning session in response to CS + presentations. Thus, the brain-stimulation US induced asymmetrical changes in the ipsi- and contralateral hemispheres; after paired training, these changes also appeared in response to $\mathrm{CS}+$ presentations.

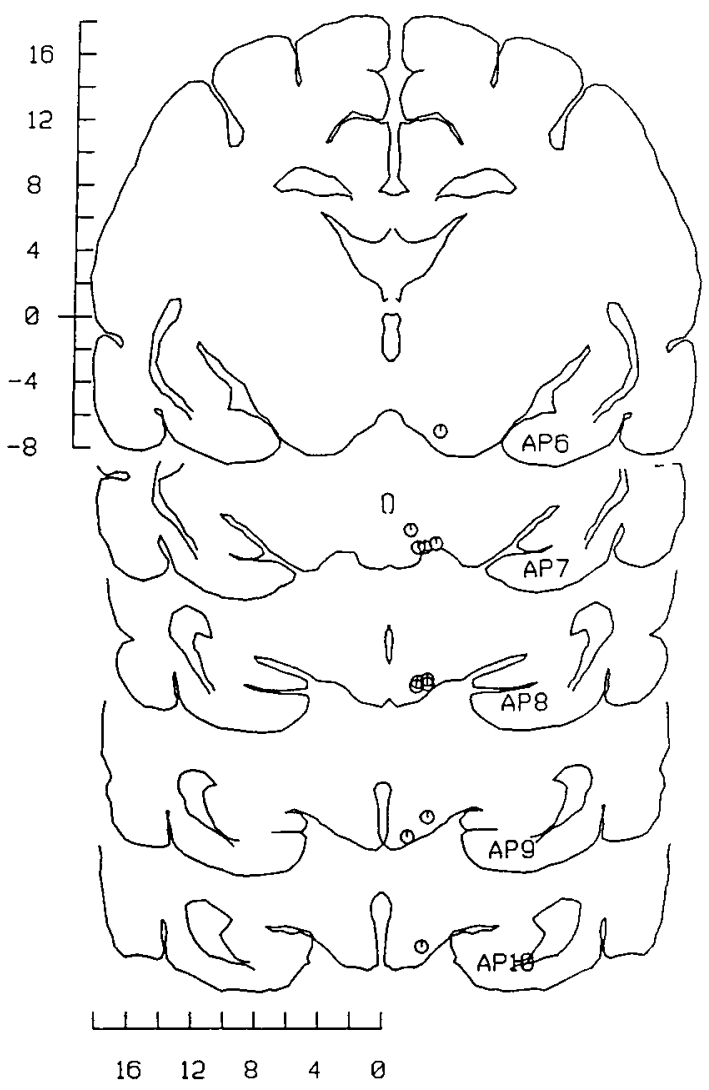

Figure 5. Locations of MFB stimulation electrodes. Circles indicate stimulation sites. Scale is in millimeters.

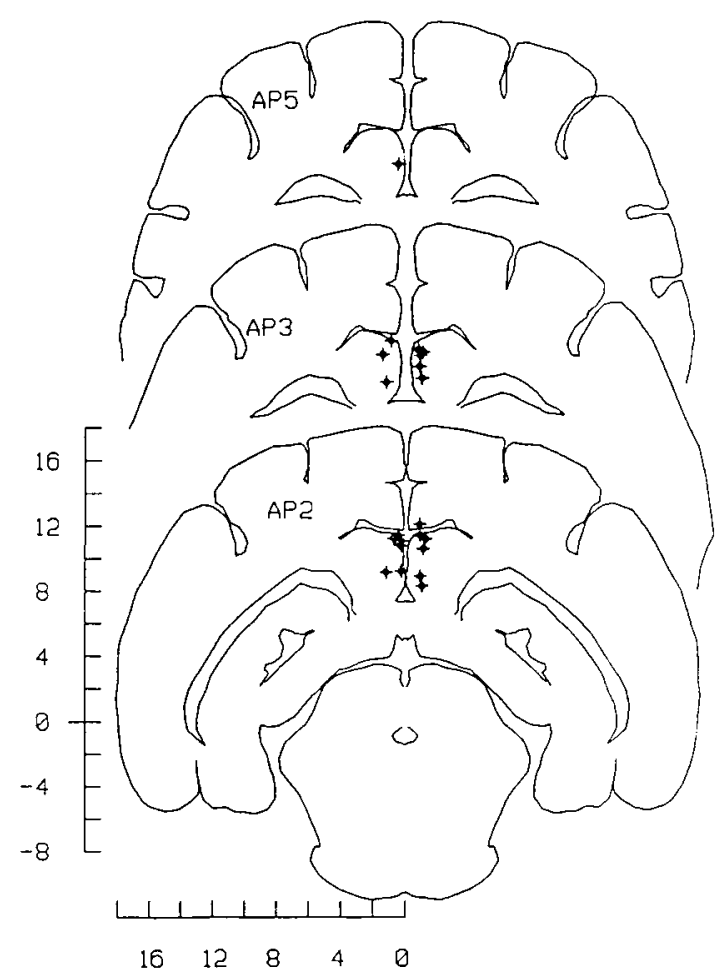

Figure 6. Localization of recording electrodes in the cingulate cortex. Each cat had one recording electrode in the left and one in the right cingulate cortex. Crosses indicate recording sites. Scale is in millimeters.

\section{Histology}

Figure 5 shows the location of the electrical stimulation electrodes. The most rostral MFB electrodes were located at the level of the lateral hypothalamus, and the most caudal electrodes were located at the level of the ventral tegmental area. Figure 6 summarizes the locations of the recording electrodes in the cingulate cortex. The electrodes were found in all the cingulate cortex layers and were approximately symmetrically located in the two sides.

\section{DISCUSSION}

\section{Conditioned Orienting Head Turns}

The cats in this study showed differential conditioning when the CS + tone was paired with unilateral electrical stimulation of the MFB and when the CS - tone was not. This differentiation appeared as head-turn CRs that were more extended, of greater acceleration, and of shorter onset latency to the CS + than to the CS - . In some cats, the head-turn CRs were related to the orienting head turns observed during the CS test session, which implies that orientation preferences were intensified during conditioning. Thus, 3 cats that predominantly turned their heads toward the direction of delivery of the tone during conditioning had already shown the same tendency during the CS test session. Furthermore, 2 other cats that predom- 
inantly made ipsilateral turns to the side of the US during conditioning had already exhibited the same tendency during the first trials of the CS test session. Even though it is not known why the latter 2 animals showed a unilateral orienting preference during the habituation session, this preference clearly defined the direction of the CR.

Eight out of 13 cats showed head-turn CRs that were not directly related to the orienting head movements observed during the CS test session. Although these cats predominantly turned their heads toward the tone during the CS test session, their CR was a head turn in one direction during conditioning. The CR was, therefore, not only an intensification of an orienting response toward the tone source. Since habituation occurred equally to both ears during the CS test session, initial preferences in orienting ipsi- or contralaterally to the side receiving the US were not responsible for the unilateral CRs. Furthermore, since no URs similar to the CRs were observed in response to the MFB stimulation, the CRs resulted from a selective enhancement of the orienting head turns in favor of one direction only. Because the CS + and CS - tones were presented alternatively to the left and the right ear in random order, the contralateral head-turn CR acquisition may primarily have occurred on those trials when the CS+ was presented to the ear contralateral to the US. Since the neuronal effects of a sound presented to one ear are larger in contralateral auditory structures (Masterton \& Imig, 1984; Phillips \& Brugge, 1985), and by inference also in nonauditory structures, it is therefore suggested that conditioning was greatest when the neuronal effects of the CS + presentation and unilateral MFB stimulation US converged-that is, when they were on the same side of the brain.

The present head-turn CR observations focus attention on the distinction proposed by Konorski (1967), and more specifically analyzed by Wagner and Brandon (1989), that USs have both specific and nonspecific components and that for some CRs the specific properties of the US are more apparent than for some others. The specific CRs, such as the rabbit nictitating membrane response (Thompson, 1986), demonstrate the primary contribution of the more specific sensory properties of the US on the CR. The nonspecific CRs, such as the cat pupillary response (Weinberger \& Diamond, 1988), rat fear potentiated startle response (Davis, 1992), rabbit heart rate response (Kapp, Markgraf, Wilson, Pascoe, \& Supple, 1991), and rat blood pressure response (LeDoux, Iwata, Pearl, \& Reis, 1986), represent the influence of more general emotional properties of the US on the CR.

The consistent observation of the present study was that the specific behavioral components of the US were not related to CR formation. On the contrary, head-turn CRs represented an intensification of orienting head turns toward the tone source in 3 cats and an intensification of an orienting preference in one direction in 2 other cats. Also, the interaction between the neuronal effects of the asymmetric CS + and the asymmetric MFB stimulation US contributed to the $\mathrm{CR}$ formation in those cats assuming a head-turn CR contralateral to the side of the US presentation. Therefore, it appears that the classification of CRs into specific and nonspecific in relation to the US may not offer an exhaustive description for CR formation during classical conditioning (cf. Lavond et al., 1993).

While the nonspecific properties of the US also contributed to the acquisition of the head-turn CR, it was nonetheless different from the acquisition of the common nonspecific CRs, such as pupillary, startle, and autonomic responses. Specifically, to our knowledge, obvious latency changes have not been reported for nonspecific CRs; however, in the present study, head-turn CRs showed a substantial reduction in onset latency over conditioning, and the onset latencies differed for the two CSs. Furthermore, nonspecific CRs appear to be acquired within a few trials, whereas specific CRs require a considerable number of trials to develop (Lennartz \& Weinberger, 1992). An asymptotic CR amplitude was reached in the present study in the third conditioning session, which suggests an acquisition rate similar to that of specific CRs. Finally, the fact that contralateral head-turn CR acquisition may have primarily occurred on those trials when the CS + was presented to the ear contralateral to the US suggests that an interaction between the specific CS and the nonspecific US can importantly contribute to learning. Thus, the study of conditioned orienting responses toward specific CSs may offer an important alternative approach in understanding the diversity of learned behaviors (Buzsaki, 1982; Holland, 1990).

\section{Conditioned Lateralized Approach \\ Response Initiation}

The present study showed selective increases in cingulate cortex evoked potentials in response to the CS + relative to the CS - . The results are thus in line with previous observations of the involvement of the cingulate cortex in differential avoidance conditioning in rabbits (see review in Gabriel, Kubota, \& Shenker, 1988). On the basis of evidence obtained from multiple-unit activity recordings with selective deafferentation of recording targets, Gabriel and his associates have proposed a limbic interaction model to explain CR initiation (Stolar et al., 1989). This model proposes that, through its reciprocal connections with the hippocampus and the limbic thalamic nuclei, the cingulate cortex forms a triggering system for avoidance $C R$ initiation. The cingulate cortex is thought to be involved in response initiation by its connections to such premotor systems as the striatum, superior colliculus, subthalamic nuclei and pontine nuclei, ultimately activating the midbrain locomotor area (Mogenson, 1987; Willner, Ahlenius, Muscat, \& Scheel-Krueger, 1991).

Although the present studies do not necessary directly support the CR initiation model of Gabriel, the results are not only compatible with that model but also suggest that it could be extended to conditioned approach movement initiation. Importantly, as response initiation in a specific direction by turning may be an integral part of approach CR initiation, it is of significance that proposed limbic 
projections to the subcortical premotor systems include as targets many structures that have previously been implied in the control of lateral eye, head, and body movementsthat is, the dorsal striatum, substantia nigra, superior colliculus, and pontine nuclei (Yeomans \& Tehovnik, 1988).

For the study of CR initiation, the present behavioral procedure is a simplification of that used in differential avoidance conditioning in rabbits (Gabriel et al., 1988). Most importantly, since the onset latency of the head-turn CR decreased considerably during conditioning and since it was substantially shorter for the CS + than for the CS-, the response initiation can be directly related to the presentation of the CS. Moreover, since, as implied by the present study, there is a tendency for head-turn CRs to be contralateral to the MFB stimulation side, and, since, as shown by a previous study (Penttonen et al., 1993), the side of CS + presentation is effective in determining the direction of the $\mathrm{CR}$, combining these two observations will allow for better control over the direction of the CR in future studies. Thus, by presenting the CSs to the ear contralateral to the MFB stimulation US, a contralateral head-turn CR can be expected to appear. Consequently, comparisons of the activations between the two sides of the cingulate cortex in relation to the side of CS and US presentations and direction of head turn should be more convenient.

\section{Cingulate Cortex Evoked Responses}

The changes in field potential responses support the possibility of selective neuronal alterations ipsilateral to the US. Increases in negative field potentials over sessions were found only in the cingulate cortex ipsilateral to the US, indicating that the paired conditioning selectively affected synaptic potentials on this side of the cingulate cortex. Furthermore, the increases in negative field potentials were greater in response to the CS + than to the CSin the ipsilateral cingulate cortex only. Although 2deoxyglycose studies have indicated that stimulation of the MFB influences neural activity on both sides of the brain (Porrino, Huston-Lyons, Bain, Sokoloff, \& Kornetsky, 1990), a greater effect has been found on the ipsilateral side (Gallistel, Gomita, Yadin, \& Campbell, 1985; Porrino et al., 1990; Yadin, Guarini, \& Gallistel, 1983). Furthermore, some lesion studies have indicated MFB stimulation to have a unilateral effect (Koob, Fray, \& Iverson, 1978; Stellar, Illes, \& Mills, 1982). The differences between the evoked potentials recorded on the two sides of the cingulate cortex exclude the possibility that the evoked potential changes resulted from diffuse, bilateral arousal effects of the US. Instead, they suggest that the greater activation of the ascending MFB pathways ipsilateral to the brain-stimulation US led to selective synaptic changes in the ipsilateral cingulate cortex in response to the $\mathrm{CS}+$.

The cross correlations that were computed between the field potential responses demonstrated that field potential waveforms also differed between the two sides of the cingulate cortex in response to $\mathrm{CS}+$ presentations. It seems quite evident that the changes in the field potentials in response to the CS + were connected to the differences between the two sides of the cingulate cortex in response to the brain-stimulation US. High correlations between the field potentials recorded in response to the $\mathrm{CS}+$ during conditioning and in response to the US during the US test session within each cingulate cortex suggest that, because the effects of the US were asymmetric in the ipsi- and contralateral cingulate cortex, the conditioned field potential changes were also asymmetric. The specificity of these effects on differential conditioning remains to be clarified, however, since there was also a correlation between $\mathrm{CS}+$ and $\mathrm{CS}-$ field potentials.

Together, the evoked potential amplitude and waveform observations suggest that the pairing of the $\mathrm{CS}+$ tone with the MFB stimulation US led to brain side specific synaptic changes in the cingulate cortex. Thus, after conditioning, the CS + presentations alone evoked local neural activations similar to those evoked during the US presentation. This effect has similarities with the activity-dependent neuromodulation observed in invertebrate Aplysia (Hawkins, Abrams, Carew, \& Kandel, 1983; Walters \& Byrne, 1983). In Aplysia, although the US is thought to activate a diffuse modulatory system that nonspecifically alters synaptic transmission, a selective alteration of synaptic efficacy occurs due to the specific temporal relation between CS + and US. It is assumed here that US field potentials reflected the effect of diffusely projecting unilateral aminergic or cholinergic systems on the cingulate cortex (e.g., Finch, Derian, \& Babb, 1984; Nieuwenhuys, Geeraedts, \& Veening, 1982; Vogt, 1985). During brain stimulation, this nonspecific MFB modulatory influence resulted in the substantial excitation of local neural populations, and thus large evoked potentials were recorded. At the start of paired conditioning, a more specific and probably less effective synaptic activation by the $\mathrm{CS}+$ presentation appeared as only minor evoked field responses. During paired conditioning, the US selectively enhanced those ipsilateral synaptic connections that were active in response to the CS + presentation. After conditioning, the CS+ alone then elicited larger ipsilateral synaptic potentials that were similar to those of the US before conditioning.

In the present study, the effect of behaviorally relevant subcortical stimulation on cingulate cortex evoked responses was demonstrated during associative conditioning. The behavioral relevance of the MFB stimulation was obvious as it induced orienting movements and exploration and as it also induced pairing-specific head-turn CRs. Similarly, long-term potentiation, generally thought to be one of the mechanisms of synaptic plasticity in the vertebrate cortex, is induced by high-frequency stimulation of hippocampal or neocortical afferent pathways (see reviews in Baudry \& Davis, 1991; Deadwyler \& Landfield, 1988). The behavioral relevance of long-term potentiation has not, however, been directly demonstrated, although similar effects of specific treatments on long-term potentiation and behavioral learning have been assumed to implicate similar mechanisms (e.g., Morris, Anderson, Lynch, 
\& Baudry, 1986). In further studies, it would be important to investigate specifically the relation between the cellular mechanisms of the field response alterations observed in the present study and those of long-term potentiation.

\section{REFERENCES}

BAUDRY, M., \& DAVIS, J. L. (EDs.). (1991). Long-term potentiation: A debate of current issues. Cambridge: MIT Press.

Berger, T. W., BassetT, J. L., \& ORR, W. B. (1991). Multiple memory systems in the mammalian brain involved in classical conditioning. In L. Dachowski \& C. F. Flaherty (Eds.), Current topics in animal learning: Brain, emotion, and cognition (pp. 271-310). Hillsdale, NJ: Erlbaum.

Buzsakı, G. (1982). The "where is it?" reflex: Autoshaping the orienting response. Journal of Experimental Analysis of Behavior, 37, 461-484.

DAvis, M. (1992). The role of the amygdala in conditioned fear. In J. P. Aggleton (Ed.), The amygdala (pp. 255-306). New York: Wiley-Liss.

DEADWYLER, S. A., \& LANDField, P. (EDs.). (1988). Long-term potentiation: From biophysics to behavior. New York: Alan R. Liss.

Finch, D. M., Derian, E. L., \& Babb, T. L. (1984). Afferent fibers to rat cingulate cortex. Experimental Neurology, 83, 468-485.

Gabriel, M., Kubota, Y., \& ShenKer, J. (1988). Limbic circuit interactions during learning. In $\mathrm{H}$. Markowitsch (Ed.), Information processing by the brain (pp. 39-63). Toronto: Hans Huber.

Gallistel, C. R., Gomita, Y., Yadin, E., \& CAmpbell, K. (1985). Forebrain origins and terminations of the medial forebrain bundle metabolically activated by rewarding stimulation or by reward-blocking doses of pimozide. Journal of Neuroscience, 5, 1246-1261.

Hawkins, R. D., Abrams, T. W., Carew, T. J., \& Kandel, E. R. (1983). A cellular mechanism of classical conditioning in Aplysia: Activity-dependent amplification of presynaptic facilitation. Science, 219, 400-405.

Holland, P. C. (1990). Forms of memory in Pavlovian conditioning. In J. L. McGaugh, N. M. Weinberger, \& G. Lynch (Eds.), Brain organization and memory: Cells, systems, and circuits (pp. 78-105). New York: Oxford University Press.

Kapp, B. S., Markgraf, C. G., Wilson, A., Pascoe, J. P., \& Supple, W. F. (1991). Contribution of the amygdala and anatomically-related structures to the acquisition and expression of aversively conditioned responses. In L. Dachowski \& C. F. Flaherty (Eds.), Current topics in animal learning: Brain, emotion, and cognition (pp. 311-346). Hillsdale, NJ: Erlbaum.

KIRK, R. E. (1968). Experimental design: Procedures for the behavioral sciences. Monterey, CA: Brooks/Cole.

KoNORSKI, J. (1967). Integrative activity of the brain. Chicago: University of Chicago Press.

KOOB, G. F., FraY, P. J., \& Iverson, S. D. (1978). Selfstimulation at the lateral hypothalamus and locus coeruleus after specific unilateral lesions of the dopamine system. Brain Research, 146, 123-140.

Korhonen, T., \& Penttonen, M. (1989a). Behavioral and neural characteristics of short-latency and long-latency conditioned responses in cats. Behavioral Neuroscience, 103, 944-955.

Korhonen, T., \& Penttonen, M. (1989b). Conditioned orienting (alpha) and delayed behavioral and evoked neural responses during classical conditioning. Behavioural Brain Research, 34, 179-197.

Lavond, D. G., KIM, J. J., \& Thompson, R. F. (1993). Mammalian brain substrates of aversive classical conditioning. Annual Review of Psychology, 44, 317-342.

LeDoux, J. E., Iwata, J., Pearl, D., \& Reis, D. J. (1986). Disruption of auditory but not visual learning by destruction of intrinsic neurons in the rat medial geniculate body. Brain Research, 371, 395-399.
LenNartz, R. C., \& Weinberger, N. M. (1992). Analysis of response systems in Pavlovian conditioning reveals rapidly versus slowly acquired conditioned responses: Support for two factors, implications for behavior and neurobiology. Psychobiology, 29, 93-119.

Masterton, R. B., \& IMIG, T. J. (1984). Neural mechanisms for sound localization. Annual Review of Physiology, 46, 275-287.

Mogenson, G. J. (1987). Limbic-motor integration. Progress in Psychobiology \& Physiological Psychology, 10, 117-170.

Morris, R. G., ANDERSON, E., LYNCH, G. S., \& BAUdRY, M. (1986). Selective impairment of learning and blockade of long-term potentiation by an N-methyl-D-aspartate receptor antagonist, AP5. Nature, 319, 774-776.

Nieuwenhuys, R., Geeraedts, L. M. G., \& Veening, J. G. (1982). The medial forebrain bundie of the rat. Journal of Comparative Neurology, 206, 49-81.

Penttonen, M., Korhonen, T., Arikoska, J., \& Hugdahl, K. (1993). Effects of lateralized US and CS presentations on conditioned head turning and bilateral cingulate cortex responses in cats. Behavioral \& Neural Biology, 59, 9-17.

Phillips, D. P., \& Brugge, J. F. (1985). Progress in neurophysiology of sound localization. Annual Review of Psychology, 36, 245-274.

Porrino, L. J., Huston-Lyons, D., Bain, G., SoKoloff, L., \& KorNETSKY, C. (1990). The distribution of changes in local cerebral energy metabolism associated with brain stimulation reward to the medial forebrain bundle of the rat. Brain Research, 511, 1-6.

SchreURS, B. G. (1989). Classical conditioning of model systems: A behavioral review. Psychobiology, 17, 145-155.

SNIDER, R. S., \& NiEMER, W. T. (1961). A stereotaxic atlas of the cat brain. Chicago: University of Chicago Press.

Stellar, J. R., Illes, J., \& Mills, L. E. (1982). Role of ipsilateral forebrain in lateral hypothalamic stimulation reward in rats. Physiology \& Behavior, 29, 1089-1097.

Stolar, N., Sparenborg, S., Donchin, E., \& Gabriel, M. (1989). Conditional stimulus probability and activity of hippocampal, cingulate cortical, and limbic thalamic neurons during avoidance conditioning in rabbits. Behavioral Neuroscience, 103, 919-934.

Thompson, R. F. (1986). The neurobiology of learning and memory. Science, 233, 941-947.

VoGT, B. A. (1985). Cingulate cortex. In A. Peters \& E. G. Jones (Eds.), Cerebral cortex (pp. 89-149). New York: Plenum Press.

WAGNER, A. R., \& BRANDON, S. E. (1989). Evolution of a structured connectionist model of Pavlovian conditioning (AESOP). In S. B. Klein \& R. R. Mowrer (Eds.), Contemporary learning theories: Pavlovian conditioning and the status of traditional learning theory (pp. 149189). Hillsdale, NJ: Erlbaum.

WALTERS, E. T., \& BYRNE, J. H. (1983). Associative conditioning of single sensory neurons suggests a cellular mechanism for learning. Science, 219, 405-408.

Weinberger, N. M., \& DiAmond, D. M. (1988). Dynamic modulation of the auditory system by associative learning. In G. M. Edelman, W. E. Gall, \& W. M. Cowan (Eds.), Auditory function: Neurobiological bases of hearing (pp. 485-512). New York: Wiley.

Willner, P., Ahlenius, S., Muscat, R., \& Scheel-Krueger, J. (1991). The mesolimbic dopamine system. In P. Willner \& J. ScheelKrueger (Eds.), The mesolimbic dopamine system: From motivation to action (pp. 3-15). New York: Wiley.

Yadin, E., GuARINI, V., \& Gallistel, C. R. (1983). Unilaterally activated systems in rats self-stimulating at sites in the medial forebrain bundle, medial prefrontal cortex, or locus coeruleus. Brain Research, 266, 39-50.

Yeomans, J. S., \& Tehovnik, E. J. (1988). Turning responses evoked by stimulation of visuomotor pathways. Brain Research, 472, 235-259.

(Manuscript received January 4, 1993; revision accepted for publication August 18, 1993.) 